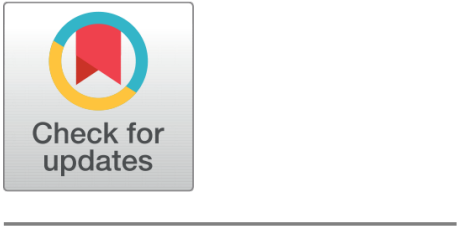

OPEN ACCESS

Received: 19.05.2021

Accepted: 15.09.2021

Published: 03.11 .2021

Citation: Debnath $\mathrm{P}$, Ishwarchandra N (2021) The Study of the Energy Conditions of the Universe and Unique Solutions of the Einstein's Field Equations in the Lights of the Theory of General Relativity. Indian Journal of Science and Technology 14(36): 2826-2831. https://doi.org/

10.17485/IJST/v14i36.873

* Corresponding author.

Tel: +91 - 7085709227

prasenjit1001@yahoo.com

Funding: None

Competing Interests: None

Copyright: (c) 2021 Debnath \& Ishwarchandra. This is an open access article distributed under the terms of the Creative Commons Attribution License, which permits unrestricted use, distribution, and reproduction in any medium, provided the original author and source are credited.

Published By Indian Society for Education and Environment (iSee)

ISSN

Print: 0974-6846

Electronic: 0974-5645

\section{The Study of the Energy Conditions of the Universe and Unique Solutions of the Einstein's Field Equations in the Lights of the Theory of General Relativity}

\author{
Prasenjit Debnath $^{1 *}$, Ngangbam Ishwarchandra ${ }^{1}$ \\ 1 The Department of Physics, National Institute of Technology Agartala, Barjala, Jirania, PO - \\ NIT Agartala, 799046, Tripura, India. Tel.: +91 - 7085709227
}

\begin{abstract}
Objectives: To study the energy conditions of the universe and to find unique solutions of the Einstein's field equations. Methods: A mathematical formulation developed to study the energy conditions of the universe and a new way of unique solutions of the famous Einstein's field equations with appropriate theoretical and mathematical analysis of the theory of general relativity. Findings: With the reference to the power series expansion of the mass function $\hat{M}(u, r)$ developed by Wang and Wu (1999), we have derived two new ways to solve Einstein's field equations by introducing new values of $n$ as $\mathrm{n}=2$ and $\mathrm{n}=-2$. Novelty: With the reference to the power series expansion of the mass function $\hat{M}(u, r)$, we have found new ways to solve Einstein's field equations with $n=2$ and $n=-2$. And we have derived a total of four new solutions of the Einstein's field equations. And the solutions are very innovative and different from (i) Schwarzschild solution and (ii) de Sitter solution. The solutions own (i) the first solution with the line element in the equation (11) describes a stationary solution. It has coordinate singularity at $r=(2 m)^{-1}$. (ii) The second solution with the line element in the equation (14) will be reduced to those Schwarzschild black holes when $m=0$ with singularities at $r=2 \mathrm{M}$. Also, it will be that dark energy when $\mathrm{M}=0$ with singularities at $r=(2 m)^{-1}$. (iii) The third solution with the line element in the equation (17) describes a stationary solution. It has coordinate singularity at $r=(2 m)^{\frac{1}{3}}$ (iv) The fourth solution with the line element in the equation (20) describes a stationary solution. It has coordinate singularity at $r=(2 m)^{\frac{1}{3}}$.
\end{abstract}

Keywords: The Mass Function; Schwarzschild Solution; Einstein's Field Equation; The Theory of General Relativity; Space - time Curvature

\section{Introduction}

A detailed study of the energy conditions of the universe and to find unique solutions of the Einstein's field equations are carried out in this paper. With the reference to the power series expansion of the mass function $\hat{M}(u, r)$, we have found new ways 
to solve Einstein's field equations with $n=2$ and $n=-2$. And we have derived a total of four new solutions of the Einstein's field equations. And the solutions are very different from(i) Schwarzschild solution (ii) de Sitter solution. The first solution with the line element describes a stationary solution. It has coordinate singularity at $r=(2 m)^{-1}$. The second solution with the line element can be reduced to those Schwarzschild black holes when $\mathrm{m}=0$ with singularities at $\mathrm{r}=2 \mathrm{M}$. Also, it will be that dark energy when $\mathrm{M}=0$ with singularities at $r=(2 m)^{-1}$. The third solution with the line element describes a stationary solution. It has coordinate singularity at $r=(2 m)^{\frac{1}{3}}$. The fourth solution with the line element describes a stationary solution. It has coordinate singularity at $r=(2 m)^{\frac{1}{3}}$.

\section{The Energy Conditions of the Universe}

To study the energy conditions of the Universe in the lights of the theory of general relativity, we begin with orthogonal null tetrad vectors such as a time - like unit vector $u^{a}$ and three space - like unit vectors $v^{a}, w^{a}, z^{a}$ such that, ${ }^{(1,2)}$

$$
\begin{gathered}
u_{a}=\frac{1}{\sqrt{2}}\left(l_{a}+n_{a}\right) \\
v_{a}=\frac{1}{\sqrt{2}}\left(l_{a}-n_{a}\right) \\
w_{a}=\frac{1}{\sqrt{2}}\left(m_{a}+\bar{m}_{a}\right) \\
z_{a}=-\frac{i}{\sqrt{2}}\left(m_{a}-\bar{m}_{a}\right)
\end{gathered}
$$

All four vectors exist with the normalization conditions $-{ }^{(3,4)}$

$$
\begin{aligned}
& u_{a} u^{a}=1, v_{a} v^{a}=w_{a} w^{a}=z_{a} z^{a}=-1 \\
& u_{a} v^{a}=u_{a} w^{a}=u_{a} z^{a}=v_{a} w^{a}=v_{a} z^{a}=w_{a} z^{a}=0
\end{aligned}
$$

Thus, the metric tensor can be written with the help of these four orthogonal tetrad vectors such as, ${ }^{(5,6)}$

$$
g_{a b}=u_{a} u_{b}-v_{a} v_{b}-w_{a} w_{b}-z_{a} z_{b}
$$

Here we are considering four velocity vectors for non - space like observers such that ${ }^{(7,8)}$

$$
U_{a}=\hat{\alpha} u_{a}+\hat{\beta} v_{a}+\hat{\gamma} w_{a}+\hat{\delta}_{a}
$$

Where, $\hat{\alpha}, \hat{\beta}, \hat{\gamma}, \hat{\delta}$ constants. The four velocity vectors $U_{a}$ based on the following conditions that can be described by ${ }^{(9,10)}$

$$
\begin{gathered}
U^{a} U_{a}=\left(\hat{\alpha} u^{a}+\hat{\beta} v^{a}+\hat{\gamma}^{a}+\hat{\delta} z^{a}\right)\left(\hat{\alpha} u_{a}+\hat{\beta} v_{a}+\hat{\gamma} w_{a}+\hat{\delta} z_{a}\right) \\
=\hat{\alpha}^{2}-\hat{\beta}^{2}-\hat{\gamma}^{2}-\hat{\delta}^{2} \geq 0
\end{gathered}
$$

For non - space like vectors,

(i) When $\hat{\beta}=\hat{\gamma}=\hat{\delta}=0, \hat{\alpha}= \pm 1$

$$
U^{a} U_{a}=\hat{\alpha}^{2}=1
$$

(ii) When $\hat{\gamma}=\hat{\delta}=0, \hat{\alpha}=\hat{\beta}= \pm 1$

$$
U^{a} U_{a}=\hat{\alpha}^{2}-\hat{\beta}^{2}=0
$$

(iii) When $\hat{\beta}=\hat{\delta}=0, \hat{\alpha}=\hat{\gamma}^{2}= \pm 1$

$$
U^{a} U_{a}=\hat{\alpha}^{2}-\hat{\gamma}^{2}=0
$$


(iv) When $\hat{\beta}=\hat{\gamma}=0, \hat{\alpha}=\hat{\delta}^{2}= \pm 1$

$$
U^{a} U_{a}=\hat{\alpha}^{2}-\hat{\delta}^{2}=0
$$

Thus, we summarize that the above conditions can be written as

$$
\begin{array}{r}
\text { (i) } \widehat{\beta}=\widehat{\gamma}=\widehat{\delta}=0, \widehat{\alpha}= \pm 1, U^{a} U_{a}=1 \\
\text { (ii) } \widehat{\gamma}=\widehat{\delta}=0, \widehat{\alpha}=\widehat{\beta}= \pm 1, U^{a} U_{a}=0 \\
\text { (iii) } \widehat{\beta}=\widehat{\delta}=0, \widehat{\alpha}=\widehat{\gamma}^{2}= \pm 1, U^{a} U_{a}=0 \\
\text { (iv) } \widehat{\beta}=\widehat{\gamma}=0, \widehat{\alpha}=\widehat{\delta}^{2}= \pm 1, U^{a} U_{a}=0
\end{array}
$$

\section{The New Solutions of the Einstein's Field Equations}

In this paper, we will present a class of exact solutions, stationary and non - stationary for the Einstein's field equations which describe non - empty, conformally flat space - time. We will also study the very physical properties of the solutions.

Wang and $\mathrm{Wu}(1999)$ introduced a mass function $\hat{M}(u, r)$ in the general line - element expressed in the form of power series expansion with the radial coordinate $\mathrm{r}^{(11,12)}$. Recently it is found that the mass function $\hat{M}(u, r)$ plays a very very important role in generating embedded and non - embedded exact solutions of the Einstein's field equations ${ }^{(13,14)}$. The mass function $\hat{M}(u, r)$ can be expressed in the following way as power series expansion as follows:

$$
\hat{M}(u, r)=\sum_{n=-\infty}^{\infty} q_{n}(u) r^{n}
$$

Where $q_{n}(u)$ is an arbitrary function of retarded time coordinate $\mathrm{u}$. The mass function can be utilized to generate non - rotating embedded solution by choosing $q_{n}(u)$ corresponding to the number $\mathrm{n}$. In the later stage, the expression is extended to find rotating system and found the significant role in generating rotating embedded solution of the Einstein's field equations ${ }^{(15,16)}$. The meaning of the power in the expansion series as follows:

- $\mathrm{n}=0$ corresponds to the mass function $\hat{M}(u, r)$ of the vacuum Kerr family solutions such as Schwarzschild, Kerr.

- $\mathrm{n}=-1$ corresponds to the mass function $\hat{M}(u, r)$ of the charged term Kerr family solutions such as Nordstrom, Kerr Newman.

- $\mathrm{n}=1$ corresponds to the mass function $\hat{M}(u, r)$ of the global monopolies solutions.

- $\mathrm{n}=3$ corresponds to the mass function $\hat{M}(u, r)$ of the de sitter cosmological models, rotating and non - rotating solutions.

The Wang - Wu power series expansion turns out to be the most convenient form to generate new embedded and non embedded solution of the Einstein's field equation if one uses Newman - Penrose formalism ( NP formalism) ( Newman and Penrose, 1962). From the above identifications of the power $n$, we can observe that $n=2$ and $n=-2$ are not observed before, so far in the correspondence to the exact solutions of the Einstein's field equations.

This is the main aim of this paper to generate exact solutions of Einstein's field equations with $n=2$ and $n=-2$ to find the actual physical meaning and to investigate the properties of the energy momentum tensors describing the matter distributions of the space - time geometry.

It is a fact that, although there is no straightforward way of solving Einstein's field equations, but a deliberate attempt is made with $n=2$ and $n=-2$ in power series expansion developed by Wang and $\mathrm{Wu}$. It is believed that $\mathrm{n}=2$ and $\mathrm{n}=-2$ will provide exact solutions of Einstein's field equations with physical interest and reasonable good interpretations of the matter distributions in the stationary and non - stationary space - time.

But first we find the exact solutions of Einstein's field equations, then, consequently we will find the physical interpretations of the line elements by studying its properties. For this very purpose, we choose Wang - Wu function $q_{n}(u)$ as follows:

$$
q_{n}(u)=\left\{\begin{array}{l}
m \cdot \text { for } \cdot n=2 \\
0 \cdot \text { for } \cdot n \neq 2
\end{array}\right.
$$

Such that mass function takes the form

$$
M(u, r) \equiv \sum_{n=-\infty}^{\infty} q_{n}(u) r^{n}=m r^{2}
$$

https://www.indjst.org/ 
Where $\mathrm{m}$ is constant and $\mathrm{u}=\mathrm{t}-\mathrm{r}$ is retarded time coordinate. Using this mass function, we find a stationary line element.

$$
\begin{aligned}
& d s^{2}=\left(1-\frac{2}{r} m r^{2}\right) d u^{2}+2 d u d r-r^{2} d \Omega^{2} \\
& d s^{2}=(1-2 m r) d u^{2}+2 d u d r-r^{2} d \Omega^{2}
\end{aligned}
$$

Here, $\mathrm{m}$ is constant and regarded as the test particle to be present in the space - time. And it is non - zero in the matter distributions in the geometry. The line element in the equation (11) describes a stationary solution. It has coordinate singularity at $r=(2 m)^{-1}$. It is called Lorentzian Horizon. Thus, it is observed that the line element is different from (i) Schwarzschild solution having $\mathrm{n}=0$ with $g_{\text {ии }}=1-2 M / r$ and singularity at $\mathrm{r}=2 \mathrm{M}$, where $\mathrm{M}$ is the Schwarzschild mass (ii) de Sitter solution having $\mathrm{n}=3$ with $g_{\text {иu }}=1-(1 / 3) r^{2} \Lambda$ and singularity at $r_{ \pm}= \pm(3 \Lambda)^{1 / 2}$.

For deriving an embedded Schwarzschild - dark energy solution, we can consider the mass function in the power expansion series if we choose Wang - Wu function $g_{n}(u)$ as follows:

$$
q_{n}(u)=\left\{\begin{array}{l}
M \cdot \text { for } \cdot n=0 \\
m \cdot \text { for } \cdot n=2 \\
0 \cdot \text { for } \cdot n \neq 0,2
\end{array}\right.
$$

Such that mass function takes the form

$$
M(u, r) \equiv \sum_{n=-\infty}^{\infty} q_{n}(u) r^{n}=M+m r^{2}
$$

Where $\mathrm{m}$ is constant and $\mathrm{u}=\mathrm{t}-\mathrm{r}$ is retarded time coordinate. Using this mass function, we find a stationary line element.

$$
d s^{2}=\left(1-\frac{2}{r}\left(M+m r^{2}\right)\right) d u^{2}+2 d u d r-r^{2} d \Omega^{2}
$$

Here, $\mathrm{m}$ is constant and regarded as the mass of the dark energy. And it is non - zero in the dark energy distribution in the Universe. The line element in the equation (14) will be reduced to that of Schwarzschild black holes when $\mathrm{m}=0$ with singularities at $r=2 M$. Also, it will be that dark energy when $M=0$ with singularities at $r=(2 m)^{-1}$. Thus, it is also observed that the line element is different from (i) Schwarzschild solution having $\mathrm{n}=0$ with $g_{\text {ии }}=1-2 M / r$ and singularity at $\mathrm{r}=2 \mathrm{M}$, where $\mathrm{M}$ is the Schwarzschild mass (ii) de Sitter solution having $\mathrm{n}=3$ with $g_{\text {ии }}=1-(1 / 3) r^{2} \Lambda$ and singularity at $r_{ \pm}= \pm(3 \Lambda)^{1 / 2}$

Now we find the exact solutions of Einstein's field equations with $n=-2$, then, consequently we will find the physical interpretations of the line elements by studying its properties. For this very purpose, we choose Wang - Wu function $g_{n}(u)$ as follows:

$$
q_{n}(u)=\left\{\begin{array}{l}
m \cdot \text { for } \cdot n=-2 \\
0 \cdot \text { for } \cdot n \neq-2
\end{array}\right.
$$

Such that mass function takes the form

$$
M(u, r) \equiv \sum_{n=-\infty}^{\infty} q_{n}(u) r^{n}=m r^{-2}
$$

Where $\mathrm{m}$ is constant and $\mathrm{u}=\mathrm{t}-\mathrm{r}$ is retarded time coordinate. Using this mass function, we find a stationary line element.

$$
\begin{aligned}
& d s^{2}=\left(1-\frac{2}{r} m r^{-2}\right) d u^{2}+2 d u d r-r^{2} d \Omega^{2} \\
& d s^{2}=\left(1-2 m r^{-3}\right) d u^{2}+2 d u d r-r^{2} d \Omega^{2}
\end{aligned}
$$

Here, $\mathrm{m}$ is constant and regarded as the mass of the dark energy. And it is non - zero in the dark energy distribution in the Universe. The line element in the equation (14) will be reduced to that of Schwarzschild black holes when $\mathrm{m}=0$ with singularities at $r=2 M$. Also, it will be that dark energy when $M=0$ with singularities at $r=(2 m)^{-1}$. Thus, it is also observed that the line element is different from (i) Schwarzschild solution having $\mathrm{n}=0$ with $g_{u u}=1-2 M / r$ and singularity at $\mathrm{r}=2 \mathrm{M}$, where $\mathrm{M}$ is the Schwarzschild mass (ii) de Sitter solution having $\mathrm{n}=3$ with $g_{u и}=1-(1 / 3) r^{2} \Lambda$ and singularity at $r_{ \pm}= \pm(3 \Lambda)^{1 / 2}$ 
For deriving an embedded Schwarzschild - dark energy solution with $n=-2$, we can consider the mass function in the power expansion series if we choose Wang - Wu function $g_{n}(u)$ as follows:

$$
q_{n}(u)=\left\{\begin{array}{l}
M \cdot \text { for } \cdot n=0 \\
m \cdot \text { for } \cdot n=-2 \\
0 \cdot \text { for } \cdot n \neq 0,-2
\end{array}\right.
$$

Such that mass function takes the form

$$
M(u, r) \equiv \sum_{n=-\infty}^{\infty} q_{n}(u) r^{n}=M+m r^{-2}
$$

Where $\mathrm{m}$ is constant and $\mathrm{u}=\mathrm{t}-\mathrm{r}$ is retarded time coordinate. Using this mass function, we find a stationary line element.

$$
d s^{2}=\left(1-\frac{2}{r}\left(M+m r^{-2}\right)\right) d u^{2}+2 d u d r-r^{2} d \Omega^{2}
$$

Here, $\mathrm{m}$ is constant and regarded as the mass of the dark energy. And it is non - zero in the dark energy distribution in the Universe. The line element in the equation (14) will be reduced to that of Schwarzschild black holes when $\mathrm{m}=0$ with singularities at $\mathrm{r}=2 \mathrm{M}$. Also, it will be that dark energy when $\mathrm{M}=0$ with singularities at $r=(2 m)^{-1}$. Thus, it is also observed that the line element is different from (i) Schwarzschild solution having $\mathrm{n}=0$ with $g_{u u}=1-2 M / r$ and singularity at $\mathrm{r}=2 \mathrm{M}$, where $\mathrm{M}$ is the Schwarzschild mass (ii) de Sitter solution having $\mathrm{n}=3$ with $g_{u u}=1-(1 / 3) r^{2} \Lambda$ and singularity at $r_{ \pm}= \pm(3 \Lambda)^{1 / 2}$

\section{Conclusion}

With reference to the theory of general relativity, the first solution with the line element in the equation (11) describes a stationary solution. It has coordinate singularity at $r=(2 m)^{-1}$. The second solution with the line element in the equation (14) will be reduced to that of Schwarzschild black holes when $m=0$ with singularities at $r=2 \mathrm{M}$. Also it will be that dark energy when $M=0$ with singularities at $r=(2 m)^{-1}$. The third solution with the line element in the equation (17) describes a stationary solution. It has coordinate singularity at $r=(2 m)^{\frac{1}{3}}$. The fourth solution with the line element in the equation (20) describes a stationary solution. It has coordinate singularity at $r=(2 m)^{\frac{1}{3}}$. The limitations of the solution are that there are many solutions of the Einstein's field equation. So, there is not any absolute or unique solution. The proposed work can be extended for other values of to get other sets of solutions of Einstein's field equation.

\section{Acknowledgement}

We truly acknowledge Dr. Aparna Nath, Associate Professor, The Department of Physics, National Institute of Technology Agartala for providing great inspiration and tremendous motivation to complete this study. Also we are extremely thankful to the Department staff for cooperation.

\section{References}

1) Bousso R. The cosmological constant. General Relativity and Gravitation. 2008;40:607-637. Available from: https://dx.doi.org/10.1007/s10714-007-05575.

2) Cai RG, Ji JY, Soh KS. Action and entropy of black holes in spacetimes with a cosmological constant. Classical and Quantum Gravity. 1998;15(9):2783-2793. Available from: https://dx.doi.org/10.1088/0264-9381/15/9/023.

3) Chandrasekhar S. The Mathematical Theory of Black Holes. Springer Netherlands. 1984. Available from: https://doi.org/10.1007/978-94-009-6469-3_2.

4) Hawking SW, Ellis GFR. The Large Scale Structure of Space-Time. Cambridge University Press. 1973. Available from: https://doi.org/10.1017/ CBO9780511524646.

5) Ibohal N. Rotating metrics admitting non-perfect fluids. General Relativity and Gravitation. 2005;37(1):19-51. Available from: https://dx.doi.org/10. 1007/s10714-005-0002-6.

6) Ibohal N, Ishwarchandra N, Singh KY. Non-vacuum conformally flat space-times: dark energy. Astrophysics and Space Science. 2011;335(2):581-591. Available from: https://dx.doi.org/10.1007/s10509-011-0767-x.

7) Mallett RL. Radiating Vaidya metric imbedded in de Sitter space. Physical Review D. 1985;31(2):416-417. Available from: https://dx.doi.org/10.1103/ physrevd.31.416.

8) Mallett R. Pion interferometry of quark - gluon plasma. Physical Review D;33:1314-1314. Available from: https://doi.org/10.1103/PhysRevD.33.1314.

9) Newman E, Penrose R. An Approach to Gravitational Radiation by a Method of Spin Coefficients. Journal of Mathematical Physics. 1962;3(3):566-578. Available from: https://dx.doi.org/10.1063/1.1724257. 
10) IBOHAL NG. Non - stationary de Sitter cosmological models. International Journal of Modern Physics D. 2009;18(05):853-863. Available from: https://dx.doi.org/10.1142/s0218271809014807.

11) Ibohal N, Dorendro L. Non - stationary rotating black holes: Entropy and Hawking's radiation. International Journal of Modern Physics D. 2005;14(08):1373-1412. Available from: https://dx.doi.org/10.1142/s0218271805007127.

12) Ibohal N. On the variable - charged black holes embedded into de Sitter space: Hawking's radiation. International Journal of Modern Physics D. 2005;14(06):973-994. Available from: https://dx.doi.org/10.1142/s0218271805007188.

13) Ovalle J, Contreras E, Stuchlik Z. Kerr-de Sitter black hole revisited. Physical Review D. 2021;103(8):84016-84016. Available from: https://dx.doi.org/10. 1103/physrevd.103.084016.

14) Sato N. The Effect of Spacetime Curvature on Statistical Distributions. arXiv preprint. 2021. doi:arXiv:2104.09019.

15) Bini D, Esposito G. Investigating new forms of gravity-matter couplings in the gravitational field equations. Physical Review D. 2021;103(6):64030. Available from: https://dx.doi.org/10.1103/physrevd.103.064030.

16) Wang A, Wu Y. Generalized Vaidya Solutions. General Relativity and Quantum Cosmology. 1999;31(1):107-114. Available from: https://dx.doi.org/10. 1023/a:1018819521971. 